\title{
Netzwerk Dermatologie und Rheumatologie
}

Die zweite i-FemMe (Inflammation Female Medical Event Austria) in Wien erfüllte im Oktober 2016 alle Erwartungen: Praxisrelevante Themen wurden von Frauen für Frauen aufbereitet und es gab die Möglichkeit zur Vernetzung in entspannter Atmosphäre.

Prim. Dr. Gabriele Eberl, Klinikum Malcherhof, Baden, gelang es als wissenschaftliche Veranstalterin der zweiten i-FemMe wieder, gemeinsam mit Pfizer ein vielfältiges Programm zusammenzustellen. Mit Prim. Prof. Dr. Beatrix Volc-Platzer, Dermatologische Abteilung, Sozialmedizinisches Zentrum Ost - Donauspital, Wien, führte sie durch die beiden Tage, die zeigten, wie interdisziplinär Rheumatologinnen und Dermatologinnen heute arbeiten. Vorträge rund um Sexualität, Familienplanung, Psoriasis, Rehabilitation, Transition, Bewegung, Kommunikation, Migration und Biosimilars gaben einen Einblick in das breite Spektrum der fachübergreifenden Kooperation.

\section{Screeningblatt Psoriasis- Komorbiditäten}

Dr. Katharina Wippel-Slupetzky von der Hautambulanz des Gesundheitszentrums Wien-Nord der WGKK, verwies auf den engen Zusammenhang zwischen Lebensstil und Psoriasis: „Unter den Triggerfaktoren für Psoriasis sind auch viele Lebensstilfaktoren wie Rauchen, Alkoholkonsum, Übergewicht oder Stress. Gezeigt wurde auch, dass Parodontose bei Patienten mit Psoriasis signifikant häufiger ist als in der Allgemeinbevölkerung." Wippel-Slupetzky wies auch auf das breite Komorbiditätsspektrum der Psoriasis hin, das in der Betreuung unbedingt beachtet werden müsse.

Um eine umfassende Betreuung zu gewährleisten, sei eine enge interdisziplinäre Zusammenarbeit von Dermatologen, Rheumatologen, Internisten, Allgemeinmedizinern, Sportwissenschaftern und Ernährungsberatern erforderlich, betonte Wippel-Slupetz- ky. Als Hilfestellung wurde ein Erhebungsblatt von Dermatologen in $\mathrm{Zu}$ sammenarbeit mit Pfizer Österreich entworfen, das Patienten eigenständig im Wartezimmer ausfüllen können. Das Erhebungsblatt gibt einen raschen Überblick über Bereiche, die eine genauere Abklärung erfordern, und kann bei Pfizer angefordert oder unter www.pfizermed.at heruntergeladen werden.

\section{Der Transitionspass für junge Patienten}

Wenn Jugendliche mit rheumatologischen Erkrankungen aus der pädiatrischen Betreuung in die Betreuung durch Rheumatologen überwechseln, ist eine gute interdisziplinäre Zusammenarbeit sehr wichtig. Wie Prof. Dr. Kirsten Minden, Kinder- und Jugendrheumatologin in Berlin, ausführte, fallen in dieser Phase viele Patienten aus der Betreuung. Einer der wesentlichen Gründe dafür sei, dass die Betroffenen nicht darauf vorbereitet wurden, was sie in der Erwachsenen-Rheumatologie erwartet: Die Betreuung wird weniger umsorgend und verlangt weit mehr Selbstständigkeit.

Ein gemeinsam von Pädiatern und Erwachsenen-Rheumatologen gestalteterTransitionsprozess erleichtert den Übergang und trägt zu einer kontinuierlichen Therapie bei. In Deutschland und in Österreich laufen verschiedene Initiativen dazu. So entstand z.B. der Transitionspass für junge Patienten, der bei der ÖGR, der ÖGKJ und der GKJR kostenlos angefordert werden kann.

\section{Herausforderung Migration}

Auf die Ärztinnen und Ärzte kommen mit der wachsenden Zahl von Patien- ten mit Migrationshintergrund neue Herausforderungen zu. Wie Dr. Christine Binder-Fritz vom Institut für Sozialmedizin, Medizinische Universität Wien, betonte, macht die fehlende gemeinsame Sprache die Betreuung dieser Patientengruppe ohne Dolmetschdienst häufig schwierig bis unmöglich. Auch das unterschiedliche Verständnis von Krankheit und Gesundheit sowie die oft divergierende Beschreibung von körperlichen und seelischen $\mathrm{Zu}$ ständen erschweren die Behandlung. Ein internetbasierter VideodolmetschDienst (www.videodolmetschen.com) bietet rund um die Uhr auch an Sonnund Feiertagen eine Lösung für das Sprachenproblem. Doch, wie Wortmeldungen aus dem Auditorium zeigten, werden sowohl Spitäler als auch niedergelassene Ärzte mit der Finanzierung dieser Dienstleistung allein gelassen.

》) Ein unterschiedliches Verständnis von Krankheit und Gesundheit erschwert die Behandlung

Eine weitere Herausforderung sei, so Binder-Fritz, das in der europäischen Kultur vielfach sehr unterschiedliche Verhältnis von Frauen und Männern zueinander. Sie empfahl, dass Patientinnen aus anderen Kulturen möglichst von Ärztinnen betreut werden sollten und männliche Patienten von Männern, da dies die Kommunikation über gesundheitliche Probleme wesentlich erleichtere.

rheuma plus $2017 \cdot 16: 15$ DOI 10.1007/s12688-017-0101-y Online publiziert: 30. Januar 2017 C) Springer-Verlag Wien 2017
Quelle: Presseaussendung Urban \& Schenk 